\title{
X-ray Emission from Gamma-Ray Bursts
}

Filippo Frontera ${ }^{1,2}$

1 University of Ferrara, Physics Dept., Via Paradiso 12, 44100 Ferrara, Italy; frontera@fe.infn.it

2 Istituto Astrofisica Spaziale e Fisica Cosmica, INAF, Via Gobetti, 101, 40129 Bologna, Italy

frontera@bo.iasf.cnr.it

Summary. In this paper I will present and discuss some of the relevant results obtained with the Italian satellite BeppoSAX on the prompt and afterglow emission of Gamma Ray Bursts (GRBs).

\section{Introduction}

After about 30 years of mystery, the distance scale issue of Gamma Ray Bursts (GRBs) has been definitely settled thanks to the X-ray astronomy mission BeppoSAX, an Italian satellite with Dutch participation [7]. BeppoSAX not only has permitted this issue to be resolved but has also provided most of the exciting results of the last seven years in GRB astronomy. The satellite, launched on 1996 April 30, was switched off on April 29, 2002, after 6 years of operational life. The high performance of BeppoSAX for GRB studies was due to a particularly well-matched configuration of its payload, with both wide field instruments (WFIs) and narrow field telescopes (NFTs).

After the exciting results obtained on GRBs in 1997 (e.g., [10, 11, 12, $15,27,31]$ ), many other GRB events were discovered with BeppoSAX during its operational life: 1082 events were detected with the GRBM (catalog in preparation), 669 of them (corresponding to $62 \%$ ) were recognized by the onboard logic and 413 (corresponding to $38 \%$ ) were identified with the ground software. Light curves with high time resolution (up to $0.5 \mathrm{~ms}$ ) are available only for the GRBs identified by the on-board logic, while for the other ones, only 1 s ratemeters are available. Of the 1082 events, 168 (corresponding to $\sim 16 \%$ ) are short $(<2 \mathrm{~s})$ GRBs, 141 of which recognized by the on-board logic. The most outstanding results were obtained from the 51 GRBs which were simultaneously detected with the GRBM and WFCs, 37 of which were followed-up with the BeppoSAX NFTs. Gamma-ray fluence of these GRBs ranges from $1.9 \times 10^{-4} \mathrm{erg} \mathrm{cm}^{-2}$ down to $2.5 \times 10^{-7} \mathrm{erg} \mathrm{cm}^{-2}$, while their duration is longer than $2 \mathrm{~s}$ ('long GRBs'). X-ray afterglows were discovered in $\sim 90 \%$ of the followed-on GRBs. However only $\sim 50 \%$ of the followed-up GRBs are detected in the optical band and $\sim 40 \%$ in the radio band. This lower rate raises the question about the origin of the so called "dark" GRBs (i.e., GRBs with no optical counterparts). Likely some of them have origin in stellar formation regions with high mass densities, which likely absorb the 
ultraviolet radiation (in the rest frame of the GRB source). However, many of the dark GRBs could have origin in galaxies at very high redshifts $(>5)$. In these cases the darkness is due to the absorption of the optical radiation by the intergalactic hydrogen.

\section{Some Highlight Results}

I will summarize here some of the most relevant results on GRBs obtained with BeppoSAX. They concern both the prompt GRB phenomenon, the afterglow emission and the GRB environment.

\subsection{Prompt Emission Spectra}

Thanks to the broad energy band (2-700 keV) covered by WFCs plus GRBM, BeppoSAX has allowed un unbiased determination of the prompt emission spectra. We found [2] that most of the time averaged spectra are well fit, down to $2 \mathrm{keV}$, with a smoothly broken power-law proposed by Band et al. [6]. In the remaining cases a simple power-law fits the data. In many cases $(\sim 70 \%)$ an optically thin synchrotron shock model (OTSSM) [39] fits the data. A confirmation of this result comes from the recent polarization measurement of the prompt emission from GRB021206 [9].

A more constraining test of the emission mechanism is the study of the spectral evolution of the GRB prompt emission. This investigation shows $[16$, $20]$ that there is a general evolution of the spectra, from hard to soft, except for the most intense events, whose hardness either mimics the GRB time profile (e.g., GRB990123) or does not evolve with time (e.g., GRB011121) [20]. A clear example of the hard-to-soft spectral evolution is given in Fig. 1, which shows the $E F(E)$ spectra of GRB970111 in the 10 contiguous time intervals (from A to $\mathrm{J}$ ) in which we subdivided the GRB time profile. As it can be seen, the fit with an OTSSM is acceptable for the almost the entire duration, except at early times. This property has been found in several GRBs and shows that at early times some other emission mechanism (likely Inverse Compton, as discussed by Frontera et al. [16]) is at work.

\subsection{Afterglow Spectra}

In general, the late afterglow spectra, at least in the time interval during which the X-ray observations are possible, are consistent with a power law $\left(I(E) \propto E^{-\Gamma}\right)$ with a photon index $\Gamma$ distributed according to a Gaussian function with mean value $\Gamma_{m}=1.93$ and standard deviation $\sigma=0.35$ [18]. The emission mechanism is likely synchrotron radiation at least in some cases (e.g., GRB970508 [21]), but in other cases a synchrotron self Compton mechanism appears to be at work (e.g., GRB000926 [23]). This mechanism implies 


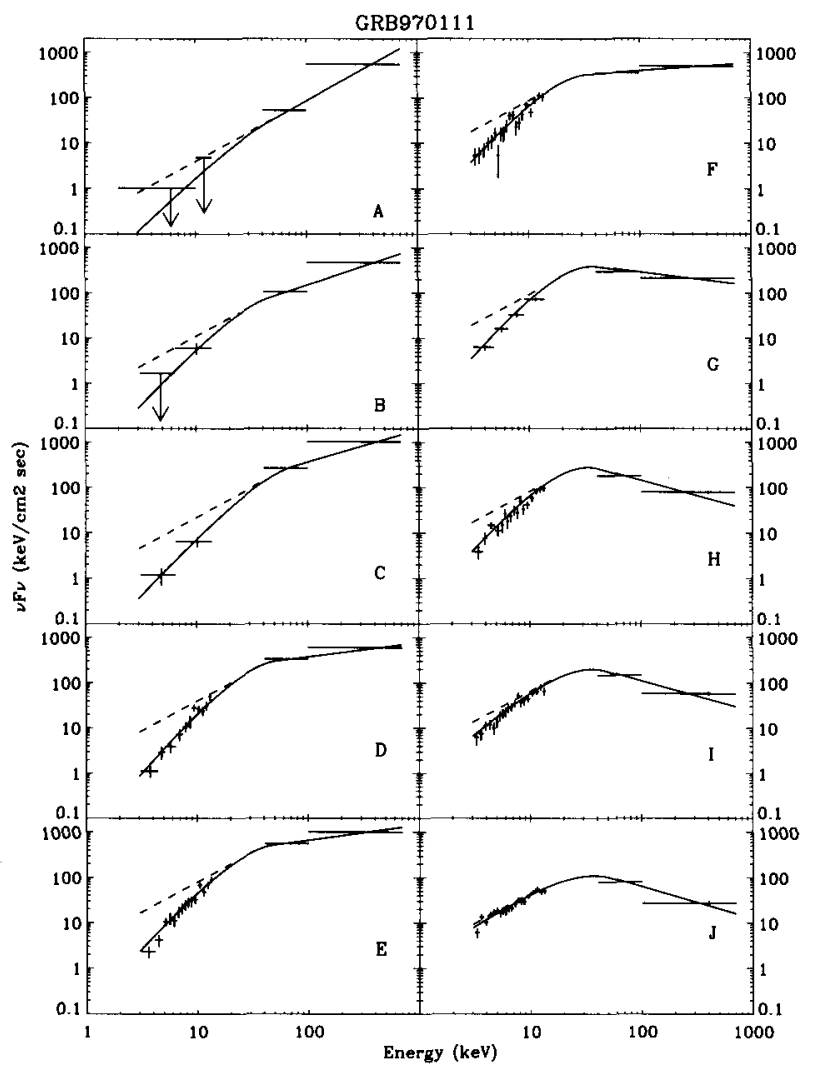

Fig. 1. $E F(E)$ spectrum of the GRB970111 prompt emission in the 10 contiguous time intervals (from A to J) in which the GRB time profile was subdivided. The GRB time duration in gamma-rays is $47 \mathrm{~s}$. The dashed line shows the maximum spectral slope which can be expected, below the energy peak, in the case of the OTSSM. The continuous line shows the best fit with a Band function. Reprinted from Frontera et al. [16].

a medium denser than a typical interstellar medium in the $\mathrm{X}$-ray production region. In the case of GRB000926, the inferred mass density is about $30 \mathrm{~cm}^{-3}$. Multiwavelength spectra, from the radio to $\mathrm{X}$-rays, are crucial to establish the emission mechanisms at work.

Recently also thermal models have been found to better fit the X-ray afterglow spectra of a few GRBs observed with the XMM-Newton and Chandra X-ray satellites: GRB001025A [41], GRB011211[34, 35], and GRB020813 [8]. In these cases an emitting plasma in collisional ionization equilibrium gives the best description of the data. 


\subsection{Fading Law of the Afterglow Emission and Its Geometry}

In general, in the time intervals in which the $\mathrm{X}$-ray observations have been possible, we find a consistency of the fading law of the X-ray afterglow emission with a single power-law $\left(F(t) \propto t^{-\delta}\right)$, with distribution of the power-law index $\delta$ consistent with a Gaussian with centroid $\delta_{m}=1.33$ and standard deviation $\sigma=0.33$ [18]. However, specially in the optical band, breaks in the afterglow light curves of several GRBs have been observed [14]. These breaks, in some cases, are also visible in the $\mathrm{X}$-ray data, if the tail of the prompt $\mathrm{X}$-ray emission is assumed to already be $\mathrm{X}$-ray afterglow emission (e.g., GRB010222 [45]). In fact this assumption has been demonstrated to be true by Frontera et al. [16], who found a correlation between X-ray fluence of the tail of the prompt emission and that of the late afterglow. The fading breaks and their time of occurrence, within the statistical uncertainties, appear to be independent of the photon energies.

The interpretation of these achromatic breaks has been discussed by various authors (e.g., $[36,37])$. In the framework of the fireball model, a break is expected to occur if the relativistically expanding material is concentrated within a cone with angular width $\theta_{c}$. As long as the Lorentz factor $\gamma$ of the out flowing material is larger than $1 / \theta_{c}$, due to relativistic beaming, the radiation is emitted within an angle $\theta_{b}=1 / \gamma$ from the cone axis, with $\theta_{b}<\theta_{c}$. When $\gamma$ drops below $1 / \theta_{c}$, the observer begins to see the edge of the cone and then the effect of the collimated outflow: a light curve steepening. Assuming a jet geometry and a uniform distribution of the energy within the jet, from the available data, Frail et al. [14] found that the distribution of the released energy per GRB is centered at $5 \times 10^{50} \mathrm{erg}$, a value almost compatible with the energy released in a supernova explosion.

From the time behavior of the X-ray afterglow light curves, we can infer only a lower limit to the jet angle. This is a few degrees, which is consistent with the lowest opening angles derived by Frail et al. [14]. A stronger constraint on the opening angle is now found by the polarization measurement of GRB021206: about 0.4 degrees [9].

\subsection{The $E_{p}$ vs. $E_{r a d}$ Relationship}

An investigation devoted to search out correlations between parameters derived from the redshift-corrected energy spectra of GRBs with known redshift has permitted us to discover [3] a power-law relation between intrinsic peak energy $E_{p}$ of the $\nu F(\nu)$ spectra and isotropic electromagnetic energy $E_{\text {rad }}$ released in the GRB event:

$$
E_{p} \propto E_{\text {rad }}^{0.52 \pm 0.06}
$$

This relation is now confirmed [4] by more BeppoSAX and HETE-2 results (see Fig. 2). It puts strong constraints to the GRB emission models: independently of the radiation pattern geometry, the $E_{p}$ vs. $E_{r a d}$ relation has to 


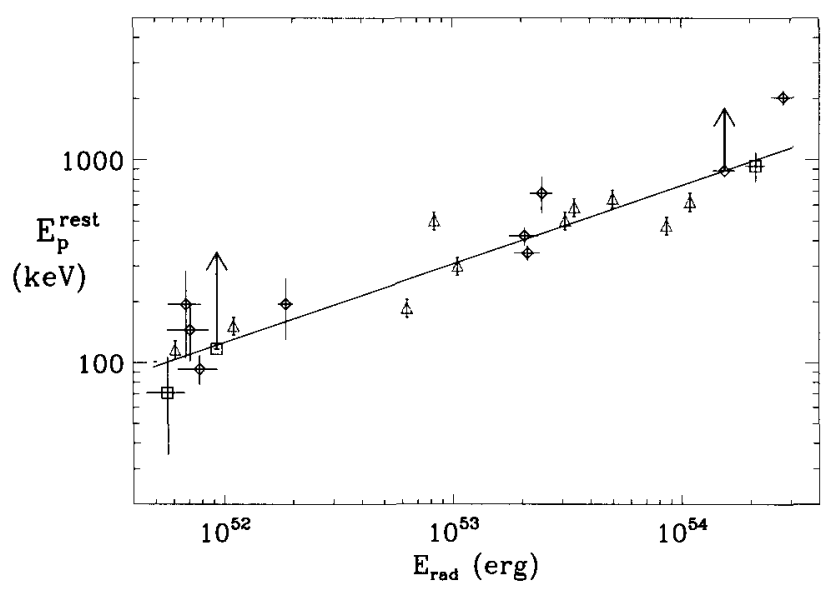

Fig. 2. Peak energy $E_{p}$ of the redshift-corrected energy $E F(E)$ spectra of GRBs with known redshift as a function of the isotropic gamma-ray energy released during prompt emission. Reprinted from Amati [4].

be satisfied. The optically thin synchrotron shock model expects a similar relation, but only with too simplified assumptions, like the same duration of all GRBs [26]. A discussion on the possible interpretations of the above relation is given by Zhang and Mészáros [46] within the internal and external shock scenario.

\subsection{The GRB Environment}

Two main probes of the GRB environment are offered by the X-ray data: detection of absorption cutoffs in the GRB continuum spectra of the prompt and/or afterglow emission, detection of emission and/or absorption spectral features. Both these probes not only give information on the circumburst environment, but are an important tool to unveil the nature of the GRB progenitors.

Time variable $N_{\mathrm{H}}$ have been discovered in the prompt emission of 4 GRBs: GRB980329 [16], GRB990705 [1], and GRB000528 [20] and GRB010214 [22]. Also in the case of the $\mathrm{X}$-ray afterglow emission, evidence of a hydrogen column density higher than that Galactic one, has been reported for GRB980329 [44], GRB980703 [40] and GRB010222 [45].

Evidence of X-ray emission features has been found in the afterglow spectra of 8 GRBs. The lines found with BeppoSAX concern 2 GRBs (970508 [32], and 000214 [5]), those found with Chandra concern GRB991216 [33] and GRB020813 [8], while those found with XMM-Newton concern GRB001025A, [41], GRB011211 [34, 35] and GRB030227 [42]. A line from GRB970828 [43] was detected with the Japanese X-ray satellite $A S C A$. Independently of the specific identification, all the detected lines point to the presence of ionized 
metals at the time of the afterglow measurements, with the ionizing radiation likely being due to the GRB power output. The relative metal abundance inferred for the lines is very large: about 10 in the case of the light metals (e.g., $[8]$ ), values $\geq 60$ in the case of Iron (e.g., [33]). Thus X-ray lines rule out the NS merger models and strongly point to an environment typical of a young supernova explosion.

Evidence of transient $\mathrm{X}$-ray absorption features in the prompt emission has been found for two events: GRB990705 [1] and GRB011211 [19]. The absorption feature from GRB990705 was interpreted by Amati et al. [1] as a cosmologically redshifted $\mathrm{K}$ edge due to neutral Fe around the GRB location, and by Lazzati et al. [25] as an absorption line due to resonant scattering of GRB photons on H-like Iron (transition $1 \mathrm{~s}-2 \mathrm{p}, E_{\text {rest }}=6.927 \mathrm{keV}$ ). In both scenarios, the observed feature points to the presence of an iron-rich environment.

In the case of the transient line feature from GRB011211, the scenario is much more complex. Given that the redshift of the GRB optical counterpart is known $(z=2.14)$, if the line feature is interpreted as due to resonant scattering of GRB photons off, e.g., H-like Ni XXVIII (rest frame energy of $8.1 \mathrm{keV}$ ), the measured line energy implies a very high blue-shift (by $0.75 \mathrm{c}$ ) of the absorbing material. A possible interpretation of the feature is discussed by Frontera et al. [19].

\subsection{GRB-SN Connection}

The conclusions of the last two sections strongly point to a connection between supernovae and GRBs. The most direct evidence of this connection has been recently obtained in the case of GRB030329/SN2003dh $[24,38]$ : nine days after the burst the optical afterglow spectrum of GRB030329 appears to be the superposition of a power-law continuum plus a spectrum consistent with that observed from Type Ic supernova SN1998bw, which was associated with GRB980425 [28] on the basis of a positional and temporal coincidence. Thus the GRB030329/SN2003dh connection has shed light also on the GRB980425/SN1998bw connection issue (see, e.g., [29] and references therein). On the basis of the results on GRB030329, also the connection of GRB980425 with SN1998bw appears strongly enforced. Also the recent $X M M-N e w t o n$ observation of SN1998bw enforces such connection. Indeed, combining the $X M M-N e w t o n$ data with the BeppoSAX data, the X-ray light curve of GRB980425/SN1998bw appears to be the superposition of two components, the GRB power-law fading emission plus the emission from a peculiar type Ic supernova [30]. 


\section{Conclusions}

In spite of the big step forward accomplished in the last 7 years after the discovery of the first X-ray afterglows, many questions about the GRB phenomenon are still open, which can only be answered with further $\mathrm{X}$-ray observations. A thorough discussion of the open issues can be found elsewhere [18]. Many of these issues are expected to be settled in the coming years, mainly with the launch of the SWIFT satellite. But a relevant contribution is expected also to be given by INTEGRAL, AGILE, and GLAST satellites, and later by the LOBSTER experiment aboard the International Space Station.

\section{References}

1. L. Amati et al. : Science 290, 953 (2000)

2. L. Amati et al. : In: Gamma-Ray Bursts in the Afterglow Era, ed. by E. Costa, F. Frontera, J. Hjorth (Springer: Berlin, Heidelberg, 2001) pp. 34-36

3. L. Amati et al. : Astron. Astrophys. 390, 81 (2002)

4. L. Amati: In: Proc. of the Frascati Workshop on Multiwavelength observations of Cosmic X-ray Sources, ed. by F. Giovannelli, L. Sabau-Graziati (Chinese J. of Astron. Astrophys., in press)

5. L.A. Antonelli et al. : Astrophys. J. Lett. 545, L39 (2000)

6. D. Band et al. : Astrophys. J. 413, 281 (1993)

7. G. Boella et al. : Astron. Astrophys. Suppl. 122, 299 (1997)

8. N.R. Butler, H.L. Marshall: astro-ph 0303539 (2003)

9. W. Coburn, S.E. Boggs: Nature 423, 415 (2003)

10. E. Costa et al. : Nature 387, 783 (1997)

11. D. Dal Fiume et al. : Astron. Astrophys. 355, 454 (2000)

12. M. Feroci et al. : Astron. Astrophys. 332, L29 (1998)

13. D.A. Frail et al. : Nature 389, 261 (1997)

14. D.A. Frail et al. : Astrophys. J. 562, L55 (2001)

15. F. Frontera et al. : Astron. Astrophys. 334, L69 (1998)

16. F. Frontera et al. : Astrophys. J. Suppl. 127, 59 (2000)

17. F. Frontera et al. : In: Gamma Ray Bursts in the Afterglow Era, ed. by E. Costa, F. Frontera, J. Hjorth (Springer: Berlin, Heidelberg, 2001) p. 106

18. F. Frontera: In: Supernovae and Gamma-Ray Bursters, ed. by K.W. Weiler (Springer: Berlin Heidelberg, 2003) pp. 317-342

19. F. Frontera et al. : Astrophys. J. submitted

20. F. Frontera et al. : In preparation

21. T.J. Galama et al. : Astrophys. J. Lett. 500, L97 (1998)

22. C. Guidorzi et al. : Astron. Astrophys. 401, 491 (2003)

23. F.A. Harrison et al. : Astrophys. J. 559, 123 (2001)

24. J. Hjorth et al. : Nature 423, 847 (2003)

25. D. Lazzati et al. : Astrophys. J. 556, 471 (2001)

26. N.M. Lloyd et al. : Astrophys. J. 534, 227 (2000)

27. M.R. Metzger et al. : Nature 387, 878 (1997)

28. F. Patat et al. : Astrophys. J. 555, 900 (2001)

29. E. Pian et al. : Astrophys. J. 536, 778 (2000) 
30. E. Pian et al. : In preparation

31. L. Piro et al. : Astron. Astrophys. 331, L41 (1998)

32. L. Piro et al. : Astrophys. J. Lett. 514, L73 (1999)

33. L. Piro et al. : Science 290, 955 (2000)

34. J.N. Reeves et al. : Nature 416, 512 (2002)

35. J.N. Reeves et al. : Astron. Astrophys. 403, 463 (2003)

36. J.E. Rhoads: Astrophys. J. Lett. 487, L1 (1997)

37. R. Sari, T. Piran, J.P. Halpern: Astrophys. J. Lett. 519, L17 (1999)

38. K.Z. Stanek.: Astrophys. J. Lett. 591, L17 (2003)

39. M. Tavani: Astrophys. J. 466, 768 (1996)

40. P.M. Vreeswijk et al. : Astrophys. J. 523, 171 (1999)

41. D. Watson et al. : Astron. Astrophys. 393, L1 (2002)

42. D. Watson et al. : Astron. Astrophys. 595, L29 (2003)

43. A. Yoshida et al. : Astron. Astrophys. Suppl. , 138433 (1999)

44. J.J.M. in't Zand et al. : Astrophys. J. Lett. 505, L119 (2001)

45. J.J.M. in't Zand et al. : Astrophys. J. 559, 710 (2001)

46. B. Zhang, P. Mészáros: Astrophys. J. 581, 1236 (2002) 